\title{
MicroRNA expression in Epstein-Barr virus-associated post-transplant smooth muscle tumours is related to leiomyomatous phenotype
}

\author{
Danny Jonigk ${ }^{1,3}$, Nicole Izykowski ${ }^{1}$, Lavinia Maegel ${ }^{1}$, Eileen Schormann ${ }^{1}$, Britta Maecker-Kolhoff ${ }^{2,3}$, \\ Florian Laenger ${ }^{1,3}$, Hans Kreipe ${ }^{1}$ and Kais Hussein ${ }^{1 *}$
}

\begin{abstract}
Epstein-Barr virus (EBV)-associated post-transplant smooth muscle tumours (PTSMT) are rare complications. In our previous molecular analysis, we have evaluated the expression of regulatory microRNA which are known to be EBV-related (miR-146a and miR-155) but found no deregulation in PTSMT. In this current analysis, we aimed to characterize the expression profiles of several hundred microRNA. Tissue samples from PTSMT and uterine leiomyomas were analysed by quantitative real-time PCR for the expression of 365 mature microRNA. PTSMT and leiomyomas share a highly similar microRNA profile, e.g. strong expression of miR-143/miR-145 cluster and low expression of miR-200c. Among EBV-related microRNA (miR-10b, miR-21, miR-29b, miR-34a, miR-127, miR-146a, miR-155, miR-200b, miR-203 and miR-429) only miR-10b and miR-203 were significantly deregulated. The expression pattern of microRNA in PTSMT is not associated with EBV infection but reflects the leiomyomatous differentiation of the tumour cells.
\end{abstract}

Keywords: Epstein-Barr virus, EBV, Post-transplant smooth muscle tumour, PTSMT, MicroRNA

\section{Introduction}

Epstein-Barr virus (EBV)-associated diseases are often associated with acquired or congenital immunosuppression or immunodeficiency, e.g. bone marrow and solid organ-transplanted patients are at a higher risk. Up to $10 \%$ of transplant recipients develop post-transplant lymphoproliferative disorders (PTLD) while EBV-associated post-transplant smooth muscle tumours (PTSMT) are rare complications $(<1 \%$ of transplant patients) [1,2]. Neoplastic spindle cells in PTSMT express leiomyogenous marker proteins such as smooth muscle actin and desmin, and the majority of tumour cells is positive for EBER. We could previously show that PTSMT differ from conventional leiomyosarcomas by their lack of marked atypia, unusual sites of involvement ( $>50 \%$ in the recipient or donor liver) and defined EBV association [2].

The molecular pathobiology of this rare neoplastic entity is not fully understood and only few experimental

\footnotetext{
* Correspondence: Hussein.Kais@MH-Hannover.de

'Institute of Pathology, Hannover Medical School, Carl-Neuberg-Str. 1,

Hannover D-30625, Germany

Full list of author information is available at the end of the article
}

analyses have addressed this issue. Ong et al. [3] have analysed cell cycle factors, cytokines and gene promoter methylation in PTSMT and found an activated mTOR/ Akt cell cycle pathway by demonstrating phosphorylated mTOR in tumour cells. In our previous analysis, we have evaluated the expression of EBV-associated human genes in PTSMT, including transcription, cell cycle and apoptosis factors and cytokines/cytokine receptors [2]. We found that the transcription factor v-myc myelocytomatosis viral oncogene homolog (avian) (MYC) is significantly upregulated in PTSMT. In addition to mRNA, we have analysed microRNAs which are known to be expressed in an EBV-related fashion (miR-146a and miR-155) but in PTSMT we found low expression levels and no delimitable deregulation. MicroRNAs are non-coding RNA molecules of 20-25 nucleotides in length $[4,5]$. These small RNA molecules can bind semi-complementarily to the 3 '-untranslated region ( $3^{\prime}$-UTR) of target mRNAs and repress translation or target mRNA for degradation. The microRNA genes can be present as single gene or gene clusters (different microRNA species are encoded on the same chromosome segment). Furthermore, microRNA families represent

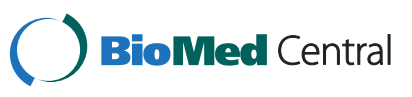


different microRNA genes with different precursor forms but very similar mature microRNA with no or minor differences in their nucleotide sequence.

Aberrant microRNA expression patterns have been identified in several neoplasms and are known to contribute to the deregulated cell homeostasis in tumour cells, e.g. leiomyomas [6-17]. In this current analysis, we aimed to characterize the expression profiles of several hundred microRNA in PTSMT, in particular regarding an association with smooth muscle phenotype and EBV infection.

\section{Material and methods}

\section{Tissue specimens}

All available PTSMT samples from our tissue archive were evaluated; these PTSMT cases have been characterized earlier [2]. Five $\mathrm{EBV}^{+}$PTSMT samples from four patients, including two tumours from one patient (\#4) were analysed (Additional file 1: Table S1). Controls: seven $\mathrm{EBV}^{-}$benign uterine leiomyomas. Formalin-fixed and paraffin-embedded (FFPE) samples were retrieved from the archives of the Institute of Pathology (Hannover Medical School/MHH, Hannover, Germany). The retrospective analysis of the samples has been approved by the local ethics committee of the Hannover Medical School (MHH).

\section{Laser microdissection of the PTSMT compartment and gene expression analysis}

Tissue from FFPE blocks with $>90 \%$ tumour cells were cut and processed for further PCR analysis. In blocks with $<90 \%$ aberrant cells, the PTSMT compartments were laser microdissected using a SmartCutPlus-System (MMI, Glattbrugg, Switzerland), as previously described [2].

A set of 365 mature microRNA and corresponding endogenous controls were analysed by quantitative real-time PCR (Pool A, Applied Biosystems, Carlsbad, CA, USA). In brief, cells were digested in proteinase $\mathrm{K}$ and RNA was extracted with phenol/chloroform $[2,18,19]$. Synthesis of cDNA from microRNA, subsequent pre-amplification of cDNA and real-time quantitative PCR with a $7900 \mathrm{HT}$ Fast Real-Time PCR system were performed according to the manufacturers' instructions (Applied Biosystems).

\section{Data analyses}

The sample- and detector-specific evaluation of amplification curves was accomplished with the software RQ Manager 1.2 (Applied Biosystems). $C_{\mathrm{T}}$ values established in this manner were converted into $\Delta C_{T}$ values and into $2^{-\Delta C T}$ values (normalized to mean of endogenous control genes). Statistical analysis was performed with Prism 5.0 (GraphPad Software, San Diego, California, USA) by applying the Mann-Whitney test for two-group comparison. $P$ values $<0.05$ were considered as statistically significant.
Cluster analysis was performed with the Qlucore Omics Explorer 2.2 (Qlucore AB, Lund, Sweden).

For target prediction, an open access bioinformatics platform was used (http://www.targetscan.org/).

\section{Results}

Similar microRNA expression profile in PTSMT and leiomyomas

Cluster analysis of the expression profile of 365 microRNA revealed that PTSMT and leiomyomas share a highly similar profile (Additional file 2: Table S2); cluster analysis could not discriminate between the two tumours.

In PTSMT, only 15/365 microRNA and in leiomyomas 23/365 microRNA showed a mean relative expression level of $>1$ (Table 1). The five microRNA with the highest expression levels in PTSMT and leiomyomas were the miR-143/miR-145 cluster (both genes are encoded on chromosome region 5q32), miR-24 (two genes on segments 9q22.32 and 19p13.13 encode for the mature miR-24 molecule), let-7b (22q13.31) and miR-21 (17q23.1). In general, most microRNA were expressed at low levels $(<1$ relative

\begin{tabular}{|c|c|c|}
\hline microRNA & PTSMT (mean) & Leiomyomas (mean) \\
\hline miR-145 & 36.82 & 36.01 \\
\hline miR-24 & 10.63 & 20.75 \\
\hline miR-143 & 7.35 & 8.12 \\
\hline let-7b & 2.61 & 2.79 \\
\hline miR-223 & 1.98 & 0.43 \\
\hline miR-342-3p & 1.96 & 1.65 \\
\hline miR-133a & 1.65 & 2.79 \\
\hline miR-19b & 1.63 & 2.23 \\
\hline miR-320 & 1.49 & 1.42 \\
\hline miR-21 & 1.34 & 5.40 \\
\hline miR-191 & 1.33 & 2.38 \\
\hline miR-30c & 1.33 & 1.23 \\
\hline miR-17 & 1.15 & 1.20 \\
\hline miR-106a & 1.14 & 1.18 \\
\hline miR-126 & 1.05 & $4.74^{* *}$ \\
\hline miR-222 & 0.75 & $7.20^{* *}$ \\
\hline miR-29a & 0.34 & $2.45^{*}$ \\
\hline miR-16 & 0.68 & 1.98 \\
\hline miR-214 & 0.11 & $1.73^{* *}$ \\
\hline miR-193b & 0.38 & $1.34^{*}$ \\
\hline let-7e & 0.19 & $1.25^{* *}$ \\
\hline miR-125b & 0.01 & 1.21 \\
\hline miR-199a-3p & 0.09 & $1.06^{* *}$ \\
\hline miR-100 & 0.01 & $1.01^{* *}$ \\
\hline
\end{tabular}


expression level) in PTSMT as well as in leiomyomas, e.g. the leiomyomatous phenotype-associated miR-150 (mean relative level of 0.38 in leiomyomas versus 0.37 in PTSMT), miR-221 (0.09 versus 0.01 ) and miR-200c ( 0.00 versus 0.01$)$.

In PTSMT, 59/365 microRNA were significantly deregulated compared with leiomyomas: a set of 51/59 microRNA were down-regulated and 8/59 were upregulated (Figure 1). However, most of these significances in down-regulated microRNA are a result of very low expression in PTSMT versus low expression in leiomyomas $(<1$ mean relative expression level), e.g. let-7c (0.22 in leiomyomas versus 0.01 in PTSMT) and miR-221 (0.09 versus 0.01). Furthermore, significantly up-regulated microRNA in PTSMT were a result of very low expression levels in leiomyomas rather than biologically relevant increased expression in PTSMT. In these 8/59 microRNA, the highest relative expression levels were less than 0.5 (miR-181a, miR-34c, miR-142-3p; Figure 1) or even less than 0.05 in PTSMT and leiomyomas (miR-138, miR-181c, miR-190, miR-330-3p/-5p, miR-504).

\section{Minor association of microRNA expression with EBV infection in PTSMT}

The following microRNA are known to be related to EBV infection in solid tumours and haematopoietic malignancies (Additional file 3: Table S3) [20-33]: miR-10b, miR-21, miR-29b, miR-34a, miR-127, miR-146a, miR-155, miR- 200b, miR-203 and miR-429. Only two of these microRNA were significantly down-regulated in $\mathrm{EBV}^{+}$PTSMT: miR$10 \mathrm{~b}$ (mean relative expression of 0.45 in leiomyomas versus 0.01 in PTSMT) and miR-203 (0.01 versus 0.00$)$. Furthermore, in leiomyomas, the level of miR-21 was higher than in PTSMT but the difference did not reach statistical significance $(5.40$ versus $1.34 ; \mathrm{p}=0.0876)$. As could be expected from our previous experiments, miR-146a and miR-155 were not deregulated while the miR-146a-homolog miR-146b was significantly down-regulated in PTSMT (mean 0.23 versus 0.73 in leiomyomas; $\mathrm{p}=0.0101$ ). Other EBV-related microRNA (miR-29b, miR-34a, miR-200b and miR-429) were expressed at similarly low levels in both leiomyomas and PTSMT (not significantly deregulated).

\section{Discussion}

\section{No EBV-related microRNA deregulation in} $\mathrm{EBV}^{+} / \mathrm{LMP}^{-}$PTSMT

In our first molecular analysis of PTSMT, we analysed the expression of miR-146a and miR-155 because these microRNA are known to be involved in the pathobiology of EBV infection of B cells [2,34,35]. In our current analysis, we could confirm our previous results and found no significant deregulation of these two microRNA using a microarray technique. Furthermore, it is remarkable that several EBV-related microRNA, other than miR146a and miR-155, are not deregulated in PTSMT. In

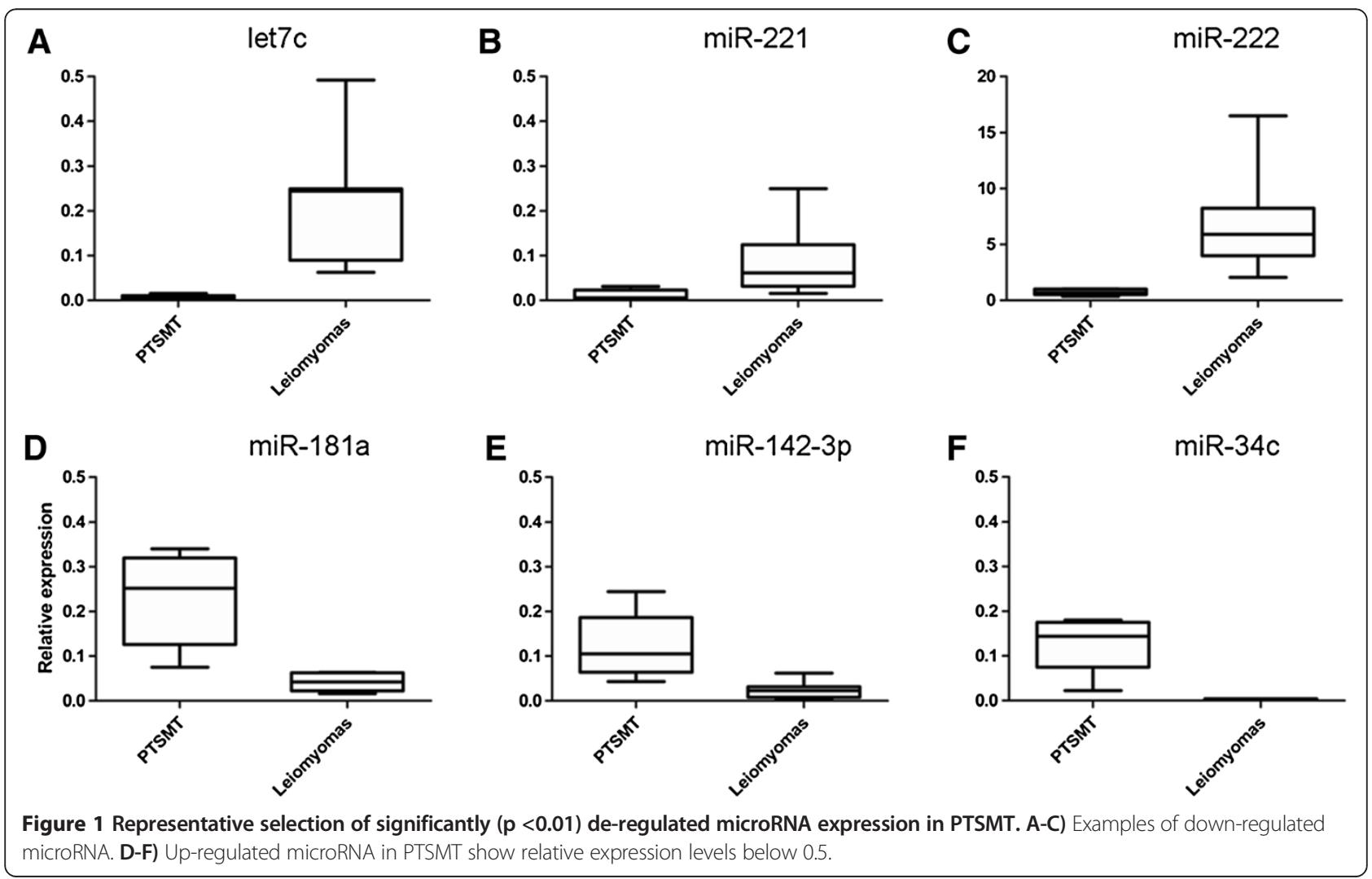


particular, miR-10b, miR-21, miR-29b, miR-34a and miR-127, which are increased in $\mathrm{EBV}^{+}$nasopharyngeal carcinoma and high grade B cell lymphomas/Burkitt lymphomas [20-33], were expressed at low or very low levels in PTSMT. In non-PTSMT EBV ${ }^{+}$carcinomas and lymphomas miR-200b, miR-203 and miR-429 are generally expressed at low levels [20-33]. In PTSMT, but also in $\mathrm{EBV}^{-}$leiomyomas, these microRNAs were also expressed at low levels, indicating no specific EBVrelated decreased expression in smooth muscle tumours. It has to be taken into account that different cell and tumour types can react in different manners to EBV infection. Furthermore, depending on the latency type, EBV induces expression of different virus proteins, which interfere in the host cell cycle. Latent membrane protein 1 (LMP1) is one of these EBV proteins and it has been shown that LMP1 alone can induce altered microRNA expression in nasopharyngeal carcinomas and lymphomas [20-33]. We and others have found previously that $\mathrm{EBV}^{+}$PTSMT express EBNA2 and EBNA3 while LMP1 expression is weak or not detectable $[2,3,36,37]$. Thus, despite EBV infection in PTSMT, our finding of no major changes in the microRNA expression profile is likely to be related to lack of LMP1 expression.

\section{Leiomyomatous phenotype-associated microRNA expression in PTSMT}

MicroRNA expression analyses in uterine leiomyomas and leiomyosarcomas were introduced only a few years ago (Additional file 4: Table S4) [6-17]. The majority of studies have evaluated patient-derived leiomyomas as a model disease of neoplastic smooth muscle proliferation. Among different studies and different analytical methods, microRNA expression patterns of patient-derived tumour samples showed a set of microRNA which are recurrently deregulated in comparison to normal uterus wall cells. Similar to PTSMT, decreased expression of miR-150, miR-200c and miR-221 and increased expression of miR21 and let-7 family members have become particularly evident in leiomyomas [6-17]. It has been demonstrated that mesenchymal cells which differentiate into smooth muscle cells in vitro change their microRNA expression patterns, e.g. down-regulation of $13 q 31.3$-clustered $\mathrm{miR}-17 / \mathrm{miR}$ $18 \mathrm{a} / \mathrm{miR}-20 \mathrm{a}$ and up-regulation of $\mathrm{miR}-181 \mathrm{a} / \mathrm{miR}-181 \mathrm{c}$ paralogs [16]. Furthermore, in smooth muscle cells, it has been shown that the 5q32-encoded miR-143/miR-145 cluster is co-expressed [38,39]. Both microRNA target a network of factors to promote vascular smooth muscle cell differentiation and repress proliferation [38,39]. We could previously demonstrate high expression of miR-143 and even higher expression of miR-145 in pulmonary vessel wall cells [40]. A similar expression pattern of high miR-143 and higher miR-145 could also be found in
PTSMT but also in leiomyomas. Because PTSMT can be found next to vessels (e.g. manifestation in cerebral sinus), it is thought that the aberrant founder cells might be derived from a vessel wall $[2,41]$. However, due to very similar miR-143/miR-145 expression patterns in PTSMT, uterus wall-derived leiomyomas and pulmonary vessels, the high expression of these two microRNA does not prove a vessel wall origin of PTSMT but reflects the smooth muscle differentiation.

In PTSMT and leiomyomas, many microRNA are expressed at low or very low levels, which makes it likely that protein translation of potential target mRNA types is not inhibited. The problem for target prediction, but simultaneously the hallmark of microRNA/mRNA biology, is the characteristic semi-complementary binding of the seven nucleotides at the 3 '-end of the mature microRNA (so-called seed sequence) to corresponding mRNA-nucleotides of the $5{ }^{\prime}$-UTR [4,5]. This semicomplementary binding is sufficient to induce a biological effect, the inhibition of mRNA/protein translation. As a result, one microRNA can bind to several $5^{\prime}$-UTR-mRNA and vice versa one $5^{\prime}$-UTR-mRNA can be targeted by several microRNA. In our previous analysis, we have evaluated the expression of several mRNA transcripts in PTSMT and leiomyomas, including MYC, vascular endothelial growth factor A (VEGFA), nuclear factor of kappa light polypeptide gene enhancer in B-cells 1 (NFKB1), tumour protein p53 (TP53), transforming growth factor, beta receptor II (TGFBR2) and transforming growth factor, beta 1 (TGFB1) [2].

Among several EBV-associated human factors, we found only MYC to be significantly increased in PTSMT [2]. The mRNA of this transcription factor can be regulated by miR-150, miR-143 and miR-145 [42,43] but no PTSMT-specific inverse correlation was found.

VEGFA can be negatively regulated by miR-200c and other microRNA. In leiomyomatous cell lines, miR-200c interaction with VEGFA has been shown [7] and accordingly, in leiomyomas as well as in PTSMT, very low levels of miR-200c correlate with increased levels of VEGFA [2].

In many different tumour types, miR-21 is aberrantly expressed, because this microRNA can target several signal networks, either directly by binding to different types of mRNA from similar signal cascades or indirectly via deregulation of factors down/up-stream to the factors directly suppressed by miR-21 [44,45]. In particular, miR-21 is a negative regulator of TP53 signalling and simultaneously a promoter of NFKB1 signalling [44,45]. Increased expression of miR-21 has been previously demonstrated in leiomyomas $[6,10,15,16]$ which we could confirm in our analysis. In smooth muscle cells, miR-21 is involved in regulation of apoptosis and TGFBR2/TGFB1 signalling [6,10,13]. TGFBR2-3'UTR has an miR-21 binding site and can therefore directly be 
regulated by miR-21 in smooth muscle cells [10]. In vitro studies also suggested an indirect regulatory interaction between miR-21 and TGFB1; of note, TGFB1 is not a direct target of miR-21 [10]. Furthermore, inhibition of miR-21 expression in smooth muscle cells indirectly increases caspase 3 and caspase 7 activity in vitro; both caspases have no miR-21 binding site $[6,13]$. The miR-21 expression was lower in PTSMT than leiomyomas but the difference was not significant. In addition, in our previous analysis we found no differences in the expression of miR-21-related NFKB1, TP53, TGFBR2 or TGFB1 between PTSMT and leiomyomas [2]. Therefore, our in situ-derived results do not reveal a PTSMT-specific deregulated miR-21 signal cascade, but an expression pattern related to smooth muscle phenotype.

Members of the let-7 family are increased in leiomyomas [15] and smooth muscle cell lines, e.g. let-7b $[13,16]$. We found that in PTSMT and leiomyomas, the strongest expressed let-7 paralog was let-7b. In leiomyomas and leiomyosarcomas, let-7c shows an inverse correlation with its target mRNA high mobility group AT-hook 2 (HMGA2) $[11,17]$. Both genes, let-7c and HMGA2, are expressed in association with size of leiomyomas [11], while in PTSMT, irrespective of the size, let-7c was almost absent.

In summary, in addition to leiomyomas and leiomyosarcomas, PTSMT is the third smooth muscle tumour type in which the microRNA expression profile could be evaluated. The expression pattern of microRNA in PTSMT is not associated with EBV infection (presumably due to lack of strong LMP1 expression) but reflects the leiomyomatous differentiation of the tumour cells.

\section{Additional files}

Additional file 1: Table S1. Characteristics of patients with PTSMT

Additional file 2: Table S2. MicroRNA expression in PTSMT in comparison to previously published data on leiomyomas (LM) and leiomyosarcomas (LMS). See Additional file 4: Table S4 for details on published data.

Additional file 3: Table S3. MicroRNA expression in association with EBV. Additional file 4: Table S4. Summary of microRNA expression data in leiomyomas and leiomyosarcomas.

\section{Competing interests}

The authors declare that they have no competing interests.

\section{Authors' contributions}

Conception of analysis (KH, DJ), histomorphology (DJ, KH, HK, FL), molecular analysis (LM, NI, ES, KH, DJ), data collection, analysis of data and manuscript preparation (DJ, FL, LM, NI, ES, BMK, HK, KH). All authors read and approved the final manuscript.

\section{Acknowledgements}

The authors thank Angelika Stucki-Koch and Regina Engelhardt for their excellent technical support and Gillian Teicke for editing the text.

\section{Funding}

DJ, FL: "Integriertes Forschungs- und Behandlungszentrum Transplantation" (IFB-Tx, German Federal Ministry of Education, reference number: 01EO0802). BMK: German Children's Cancer Fund and IFB-Tx.

\section{Author details}

'Institute of Pathology, Hannover Medical School, Carl-Neuberg-Str. 1, Hannover D-30625, Germany. ${ }^{2}$ Department of Paediatric Haematology and Oncology, Hannover Medical School, Hannover, Germany. ${ }^{3}$ Integrated Research and Treatment Center Transplantation (IFB-TX), Hannover, Germany.

Received: 25 April 2013 Accepted: 25 June 2013

Published: 6 July 2013

\section{References}

1. Shroff $R$, Rees $L:$ The post-transplant lymphoproliferative disorder - a literature review. Pediatr Nephrol 2004, 19:369-377.

2. Jonigk $D$, Laenger $F$, Maegel $L$, et al: Molecular and clinicopathological analysis of Epstein-Barr virus-associated posttransplant smooth muscle tumors. Am J Transplant 2012, 12:1908-1917.

3. Ong KW, Teo M, Lee $\mathrm{V}$, et al: Expression of EBV latent antigens, mammalian target of rapamycin, and tumor suppression genes in EBV-positive smooth muscle tumors: clinical and therapeutic implications. Clin Cancer Res 2009, 15:5350-5358.

4. Hussein K: Pathobiology of the microRNA system. Pathologe 2012, 33:70-78

5. Esteller M: Non-coding RNAs in human disease. Nat Rev Genet 2011, 12:861-874

6. Fitzgerald JB, Chennathukuzhi V, Koohestani F, Nowak RA, Christenson LK: Role of microRNA-21 and programmed cell death 4 in the pathogenesis of human uterine leiomyomas. Fertil Steril 2012, 98:726-734. e2.

7. Chuang TD, Panda H, Luo X, Chegini N: miR-200c is aberrantly expressed in leiomyomas in an ethnic-dependent manner and targets ZEBs, VEGFA, TIMP2, and FBLN5. Endocr Relat Cancer 2012, 19:541-556.

8. Georgieva B, Milev I, Minkov I, Dimitrova I, Bradford AP, Baev V: Characterization of the uterine leiomyoma microRNAome by deep sequencing. Genomics 2012, 99:275-281.

9. Zavadil J, Ye H, Liu Z, et al: Profiling and functional analyses of microRNAs and their target gene products in human uterine leiomyomas. PLoS One 2010, 5:e12362.

10. Pan Q, Luo X, Chegini N: MicroRNA 21: response to hormonal therapies and regulatory function in leiomyoma, transformed leiomyoma and leiomyosarcoma cells. Mol Hum Reprod 2010, 16:215-227.

11. Peng Y, Laser J, Shi G, et al: Antiproliferative effects by Let-7 repression of high-mobility group A2 in uterine leiomyoma. Mol Cancer Res 2008, 6:663-673.

12. Nuovo GJ, Schmittgen TD: Benign metastasizing leiomyoma of the lung: clinicopathologic, immunohistochemical, and micro-RNA analyses. Diagn Mol Pathol 2008, 17:145-150

13. Pan Q, Luo X, Chegini N: Differential expression of microRNAs in myometrium and leiomyomas and regulation by ovarian steroids. J Cell Mol Med 2008, 12:227-240.

14. Marsh EE, Lin Z, Yin P, Milad M, Chakravarti D, Bulun SE: Differential expression of microRNA species in human uterine leiomyoma versus normal myometrium. Fertil Steril 2008, 89:1771-1776.

15. Wang $T$, Zhang $X$, Obijuru $L$, et al: A micro-RNA signature associated with race, tumor size, and target gene activity in human uterine leiomyomas. Genes Chromosomes Cancer 2007, 46:336-347.

16. Danielson LS, Menendez S, Attolini CS, et al: A differentiation-based microRNA signature identifies leiomyosarcoma as a mesenchymal stem cell-related malignancy. Am J Pathol 2010, 177:908-917.

17. Shi $G$, Perle MA, Mittal $K$, et al: Let-7 repression leads to HMGA2 overexpression in uterine leiomyosarcoma. J Cell Mol Med 2009, 13:3898-3905.

18. Hussein $K$, Theophile $K$, Büsche $G$, et al: Aberrant microRNA expression pattern in myelodysplastic bone marrow cells. Leuk Res 2010, 34:1169-1174.

19. Jonigk D, Merk M, Hussein $K$, et al: Obliterative airway remodeling: molecular evidence for shared pathways in transplanted and native lungs. Am J Pathol 2011, 178:599-608.

20. Onnis A, Navari M, Antonicelli G, et al: Epstein-Barr nuclear antigen 1 induces expression of the cellular microRNA hsa-miR-127 and impairing B-cell differentiation in EBV-infected memory B cells. New insights into the pathogenesis of Burkitt lymphoma. Blood Cancer J 2012, 2:e84. 
21. Rosato P, Anastasiadou E, Garg N, et al: Differential regulation of miR-21 and miR-146a by Epstein-Barr virus-encoded EBNA2. Leukemia 2012, 26:2343-2352

22. Forte E, Salinas RE, Chang C, et al: The Epstein-Barr virus (EBV)-induced tumor suppressor microRNA MiR-34a is growth promoting in EBVinfected B cells. J Virol 2012, 86:6889-6898.

23. Yu H, Lu J, Zuo L, Yan Q, Yu Z, Li X, Huang J, Zhao L, Tang H, Luo Z, Liao Q, Zeng Z, Zhang J, Li G: Epstein-Barr virus downregulates microRNA203 through the oncoprotein latent membrane protein 1: a contribution to increased tumor incidence in epithelial cells. J Virol 2012, 86:3088-3099.

24. Du ZM, Hu LF, Wang HY, Yan LX, Zeng YX, Shao JY, Ernberg I: Upregulation of MiR-155 in nasopharyngeal carcinoma is partly driven by LMP1 and LMP2A and downregulates a negative prognostic marker JMJD1A. PLoS One 2011, 6:e19137.

25. Linnstaedt SD, Gottwein E, Skalsky RL, Luftig MA, Cullen BR: Virally induced cellular microRNA miR-155 plays a key role in B-cell immortalization by Epstein-Barr virus. J Virol 2010, 84:11670-11678.

26. Li G, Wu Z, Peng Y, et al: MicroRNA-10b induced by Epstein-Barr virusencoded latent membrane protein-1 promotes the metastasis of human nasopharyngeal carcinoma cells. Cancer Lett 2010, 299:29-36.

27. Ellis-Connell AL, lempridee T, Xu I, Mertz JE: Cellular microRNAs200b and 429 regulate the Epstein-Barr virus switch between latency and lytic replication. J Virol 2010, 84:10329-10343.

28. Lin Z, Wang X, Fewell C, Cameron J, Yin Q, Flemington EK: Differential expression of the miR-200 family microRNAs in epithelial and B cells and regulation of Epstein-Barr virus reactivation by the miR-200 family member miR-429. J Virol 2010, 84:7892-7897.

29. Shinozaki A, Sakatani T, Ushiku T, et al: Downregulation of microRNA-200 in EBV-associated gastric carcinoma. Cancer Res 2010, 70:4719-4727.

30. Yin Q, Wang X, Fewell C, et al: MicroRNAmiR-155 inhibits bone morphogenetic protein (BMP) signaling and BMP-mediated Epstein-Barr virus reactivation. J Virol 2010, 84:6318-6327.

31. Anastasiadou E, Boccellato F, Vincenti S, et al: Epstein-Barr virus encoded LMP1 downregulates TCL1 oncogene through miR-29b. Oncogene 2010, 29:1316-1328.

32. Yin Q, McBride J, Fewell C, et al: MicroRNA-155 is an Epstein-Barr virus-induced gene that modulates Epstein-Barr virus-regulated gene expression pathways. J Virol 2008, 82:5295-5306.

33. Motsch N, Pfuhl T, Mrazek J, Barth S, Grässer FA: Epstein-Barr virus-encoded latent membrane protein 1 (LMP1) induces the expression of the cellular microRNA miR-146a. RNA Biol 2007, 4:131-137.

34. Zheng ZM: Viral oncogenes, noncoding RNAs, and RNA splicing in human tumor viruses. Int J Biol Sci 2010, 6:730-755.

35. Cameron JE, Yin Q, Fewell C, et al: Epstein-Barr virus latent membrane protein 1 induces cellular MicroRNA miR-146a, a modulator of lymphocyte signaling pathways. J Virol 2008, 82:1946-1958.

36. Rogatsch $\mathrm{H}$, Bonatti $\mathrm{H}$, Menet $\mathrm{A}$, Larcher $\mathrm{C}$, Feichtinger $\mathrm{H}$, Dirnhofer S: Epstein-Barr virus-associated multicentric leiomyosarcoma in an adult patient after heart transplantation: case report and review of the literature. Am J Surg Pathol 2000, 24:614-621.

37. Rougemont AL, Alfieri C, Fabre M, et al: Atypical Epstein-Barr virus (EBV) latent protein expression in EBV-associated smooth muscle tumours occurring in paediatric transplant recipients. Histopathology 2008, 53:363-367.

38. Cheng Y, Liu X, Yang J, et al: MicroRNA-145, a novel smooth muscle cell phenotypic marker and modulator, controls vascular neointimal lesion formation. Circ Res 2009, 105:158-166.

39. Cordes KR, Sheehy NT, White MP, et al: miR-145 and miR-143 regulate smooth muscle cell fate and plasticity. Nature 2009, 460:705-710.

40. Bockmeyer $C L$, Maegel $L$, Janciauskiene $S$, et al: Plexiform vasculopathy of severe pulmonary arterial hypertension and microRNA expression. J Heart Lung Transplant 2012, 31:764-772.

41. Chaves NJ, Kotsimbos TC, Warren MA, et al: Cranial leiomyosarcoma in an Epstein-Barr virus (EBV)-mismatched lung transplant recipient. J Heart Lung Transplant 2007, 26:753-755.

42. Jiang $X$, Huang $H$, Li Z, et al: Blockade of miR-150 Maturation by MLL-Fusion/MYC/LIN-28 Is Required for MLL-Associated Leukemia. Cancer Cell 2012, 22:524-535.
43. Huang S, Guo W, Tang Y, Ren D, Zou X, Peng X: miR-143 and miR-145 inhibit stem cell characteristics of PC-3 prostate cancer cells. Oncol Rep 2012, 28:1831-1837.

44. Buscaglia LE, Li Y: Apoptosis and the target genes of microRNA-21. Chin J Cancer 2011, 30:371-380.

45. Jazbutyte $V$, Thum T: MicroRNA-21: from cancer to cardiovascular disease. Curr Drug Targets 2010, 11:926-935.

doi:10.1186/2045-3329-3-9

Cite this article as: Jonigk et al:: MicroRNA expression in Epstein-Barr virus-associated post-transplant smooth muscle tumours is related to leiomyomatous phenotype. Clinical Sarcoma Research 2013 3:9.

\section{Submit your next manuscript to BioMed Central and take full advantage of:}

- Convenient online submission

- Thorough peer review

- No space constraints or color figure charges

- Immediate publication on acceptance

- Inclusion in PubMed, CAS, Scopus and Google Scholar

- Research which is freely available for redistribution

Submit your manuscript at www.biomedcentral.com/submit
C) BioMed Central 